Enfance en difficulté

\title{
L'intervention en contexte de cyberintimidation : les pratiques des enseignants
}

\author{
Émilie Myriam Roy et Claire Beaumont
}

Volume 2, mars 2013

URI : https://id.erudit.org/iderudit/1016248ar

DOI : https://doi.org/10.7202/1016248ar

Aller au sommaire du numéro

\section{Éditeur(s)}

Université Laurentienne

ISSN

1920-6275 (imprimé)

1929-8544 (numérique)

Découvrir la revue

Citer cet article

Roy, É. M. \& Beaumont, C. (2013). L’intervention en contexte de cyberintimidation : les pratiques des enseignants. Enfance en difficulté, 2, 85-109. https://doi.org/10.7202/1016248ar

\section{Résumé de l'article}

La cyberintimidation est une forme de violence de plus en plus préoccupante pour la société et pour le milieu scolaire. Souvent commises dans Internet ou sur le téléphone cellulaire, les agressions ont des conséquences psychologiques, sociales et scolaires très graves chez les victimes, les agresseurs et les témoins, et il est important d'intervenir adéquatement. Cette étude vise à mieux connaître les types d'intervention utilisés par les enseignants auprès des élèves qui ont des comportements de cyberintimidation et à mieux comprendre les bases qui expliquent ces choix d'intervention. Des entrevues menées auprès de 10 enseignants des $7^{\mathrm{e}}, 8^{\mathrm{e}}$ et $9^{\mathrm{e}}$ années ont permis de relever leurs interventions les plus courantes pour gérer et traiter les comportements de cyberintimidation de leurs élèves, connaître leur perception de l'efficacité de ces pratiques et explorer les fondements théoriques sur lesquels ils s'appuient dans leurs choix d'intervention. L'analyse de leurs témoignages, mise en perspective avec la littérature scientifique sur le sujet, permet de proposer un nouveau modèle d'intervention auprès des auteurs de cyberintimidation. Après avoir confronté les résultats au modèle d'intervention d'Hinduja et Patchin (2009), les auteures suggèrent de nouvelles pistes d'intervention pour améliorer la formation des enseignants dans ce domaine. 
www.laurentienne.ca

\title{
L'intervention en contexte de cyberintimidation : les pratiques des enseignants
}

\author{
Émilie Myriam Roy et Claire Beaumont \\ Université Laval
}

\section{Introduction}

Cet article présente les résultats d'une recherche qui permet de mieux comprendre comment les enseignants interviennent auprès des jeunes qui ont des comportements de cyberintimidation. Il traite plus précisément des actions posées par les enseignants auprès de ces jeunes, des interventions jugées efficaces et, finalement, des fondements théoriques sur lesquels se basent les enseignants pour choisir leurs interventions.

La cyberintimidation est une forme d'intimidation à laquelle de plus en plus de jeunes sont exposés. Les écrits scientifiques sur le sujet se développent de façon extrêmement rapide et on peut maintenant mieux comprendre le phénomène. La recherche nous informe que cette nouvelle forme de violence, impliquant l'utilisation des technologies de l'information et de la communication pour faire du tort à une autre personne, est plus présente chez les jeunes de 12 à 15 ans et les agresseurs la commettent surtout quand ils sont chez eux (Cassidy, Jackson, \& Brown, 2009; Dehue, Bolman, \& Völlink, 2008; Slonje \& Smith, 2008). Cependant, puisque la cyberintimidation prend généralement ses origines dans des situations d'intimidation vécues à l'école (Erdur-Baker, 2010), les milieux scolaires participent à la recherche de stratégies de prévention et d'intervention efficaces pour la réduire. 
Selon Cassidy et al. (2009), la cyberintimidation peut prendre la forme de messages blessants, vulgaires, moqueurs ou menaçants (textes, photos ou vidéos) diffusés par Internet ou la téléphonie cellulaire. Plusieurs auteurs définissent la cyberintimidation comme une forme d'agression intentionnelle et répétitive perpétrée à l'aide des nouvelles technologies (Smith et al., 2008; Vandebosch \& van Cleemput,2008).Vandebosch et van Cleemput (2008) soulignent toutefois qu'un seul acte négatif dans Internet peut être considéré comme un acte de cyberintimidation s'il est précédé d'intimidation dite traditionnelle, soit lorsque l'agresseur et la victime se côtoient physiquement. Pour Prud'homme (2008) et Hinduja et Patchin (2009), l'inégalité dans le rapport de forces, caractéristique généralement mentionnée dans l'intimidation traditionnelle, n'existe pas nécessairement en situation de cyberintimidation. L'utilisation des technologies pour intimider ou causer du tort à autrui fait donc en sorte que chacun, fort ou faible, peut devenir agresseur ou victime. Prudents, Vandebosch et van Cleemput (2008) rappellent qu'une situation de conflit n'en est pas toujours une de cyberintimidation, celle-ci ayant lieu quand la victime vit une forme de vulnérabilité par rapport à son agresseur. Toujours selon ces auteurs, les risques de mésinterprétation - où le message sans malice d'un expéditeur est reçu comme une insulte par son destinataire, par exemple - complexifient l'identification des cas d'intimidation. Cette complexité entourant la définition et l'identification de la cyberintimidation ne rend pas le problème moins réel. Calvete, Orue, Villardón et Padilla (2010) suggèrent d'ailleurs que $44,1 \%$ des jeunes âgés entre 12 et 17 auraient déjà commis des actes de cyberintimidation.

La liste de caractéristiques de la cyberintimidation que proposent Hinduja et Patchin (2009) explique la difficulté à freiner le phénomène. D'abord, la rapidité de la diffusion des contenus méprisants et le grand bassin de récipiendaires potentiels font en sorte qu'il est difficile d'en effacer toutes les traces. Les conséquences de la cyberintimidation sont alors plus difficiles à réparer puisque les agressions se perpétuent dans le temps et le cyberespace. De plus, la cyberintimidation peut survenir à tout moment et en tout lieu. La victime n'a donc pas la possibilité de trouver une certaine sécurité, un répit, en s'éloignant physiquement de l'agresseur.

Selon Cassidy et al. (2009), les raisons qui motivent les cyberagresseurs à passer à l'acte sont, par ordre d'importance, 1) l'hostilité envers la victime; 2 ) le désir de vengeance; 3 ) l'envie de faire comme leurs amis, et 4) le simple plaisir. Selon Walrave et Heirman (2011), 
les jeunes qui publient beaucoup d'informations personnelles en ligne ou tentent de se lier d'amitié avec des personnes un peu plus âgées augmentent leurs risques d'être la cible d'une cyberagression. Par ailleurs, puisque les agresseurs peuvent cacher leur identité (Dehue et al., 2008), certaines victimes d'intimidation traditionnelle ou de cyberintimidation peuvent être à leur tour tentées de recourir, de façon anonyme, à la cyberintimidation pour se venger de leur agresseur (König, Gollwitzer, \& Steffgen, 2010; Vandebosch \& van Cleemput, 2008; Walrave \& Heirman, 2011). Des auteurs mentionnent aussi que les témoins de situation d'intimidation en ligne sont également plus à risque de devenir agresseurs, soit par l'apprentissage de comportements observés, mais surtout par crainte d'être associés à la victime et de subir le même sort (Conway \& McCord, 2002; Suzuki \& Lucas, 2010). De plus, selon Jones, Manstead et Livingstone (2011), ces jeunes s'identifiant à un groupe agresseur pourraient voir leurs comportements guidés par la volonté du groupe plutôt que leur volonté personnelle, diminuant ainsi leurs sentiments de honte et de responsabilité à l'égard du tort causé à la victime.

En plus de cet effet d'entraînement, la cyberintimidation fournit une impression d'anonymat qui contribue, selon Ang et Goh (2010), à rendre les agresseurs encore plus insensibles aux sentiments des victimes qu'en situation d'intimidation traditionnelle. La distance physique séparant les agresseurs de leurs victimes peut faire en sorte que les premiers soient à l'abri des réactions émotionnelles et physiques immédiates de leurs victimes, accentuant ainsi la gravité de leurs propos ou de leurs actes. De plus, en cachant leur identité réelle, les agresseurs se libèrent des pressions sociales, morales et éthiques qui incitent à se comporter selon des normes acceptées.

\section{Les conséquences de la cyberintimidation}

De nombreux auteurs ont relevé les conséquences psychologiques, sociales et scolaires de la cyberintimidation chez les victimes et les agresseurs. Cassidy et al. (2009) et O'Brennan, Bradshaw et Sawyer (2009) suggèrent que les agressions en ligne provoquent chez leurs victimes un haut niveau d'anxiété, la crainte de se rendre à l'école et des difficultés de concentration. À plus long terme, et même après que les agressions ont cessé, une réputation souillée et un manque de confiance et d'estime de soi résultant de cyberintimidation peuvent affecter les habiletés des victimes à se faire des amis et leur causer du 
stress, des symptômes de dépression et des pensées suicidaires. Klomek, Sourander et Gould (2010) et Hinduja et Patchin (2010) ajoutent que non seulement les victimes, mais les cyberagresseurs eux-mêmes seraient à risque d'avoir des pensées ou des comportements suicidaires. On a aussi établi des liens étroits entre les comportements de cyberintimidation et la faible estime de soi, les difficultés sociales, les problèmes de conduite et la dépendance à la cigarette et à l'alcool (Hinduja \& Patchin, 2010; Sourander et al., 2010). La gravité des conséquences de cette nouvelle forme de violence invite les chercheurs à en étudier les différentes facettes pour pouvoir mieux orienter le personnel scolaire vers des interventions éducatives appropriées (Beaumont, 2009; Shariff, 2005).

\section{Les interventions efficaces contre la cyberintimidation : recension des écrits}

À la lumière de leurs nombreux travaux portant sur la cyberintimidation, Hinduja et Patchin (2009) ont suggéré un modèle d'intervention sous la forme d'un continuum d'interventions appropriées pour faire diminuer les comportements de cyberintimidation. Ces auteurs ont classé les comportements de cyberintimidation en trois catégories de gravité, soit 1) mineure (traiter de noms, ignorer), 2) modérée (répandre des rumeurs, afficher des photos ou vidéos sans permission), et 3) sévère (menacer, harceler). Pour répondre à des agressions de gravité mineure, ils suggèrent, par exemple, d'appliquer des sanctions créatives aux agresseurs (réalisation de projets scolaires, conception d'affiches) ou encore d'organiser des rencontres avec les parents ou un professionnel (travailleur social, psychologue, etc.) pour signaler à l'élève que son comportement est inacceptable et qu'il ne sera pas toléré. Pour intervenir contre les agressions de gravité modérée, ils proposent plutôt une rencontre avec la direction de l'établissement, un plan d'intervention concernant l'apprentissage de comportements plus appropriés ou des sanctions telles qu'une "retenue ». En ce qui concerne les cas sévères, ils recommandent des actions en justice ou l'expulsion de l'élève de l'établissement scolaire (voir Tableau 1).

Nous avons mis à profit les écrits d'autres chercheurs et théoriciens pour expliquer comment les interventions conçues en fonction du modèle théorique de Hinduja et Patchin (2009) peuvent modifier des comportements non désirés. D'abord, Hinduja et Patchin considèrent que l'implication parentale, l'autorité scolaire et la participation des pairs à des projets créatifs sont toutes des pistes à considérer pour aider les jeunes cyberintimidateurs à modifier leurs comportements. 


\section{Tableau 1 - Modèle d'intervention selon Hinduja \& Patchin} (2009)

\section{Gravité du geste}

\begin{tabular}{|l|l|l|}
\hline Gravité mineure & Gravité modérée & Gravité sévère \\
\hline - Taquiner & - Nuire à la & - Menacer (agression \\
- Ignorer & réputation & physique ou mort) \\
- Traiter de noms & - Répandre des & - Harceler (traquer) \\
- Se moquer & rumeurs & - Intimider \\
- Ridiculiser, rabaisser & Afficher des & \\
& photos sans & \\
& permission & \\
& - Créer des vidéos & \\
& intimidantes & \\
\hline
\end{tabular}

Interventions à mener selon la gravité du geste posé

\begin{tabular}{|l|l|l|}
\hline $\begin{array}{l}\text { Rencontre avec les } \\
\text { parents }\end{array}$ & $\begin{array}{l}\text { Rencontre avec } \\
\text { le directeur ou la } \\
\text { directrice }\end{array}$ & $\begin{array}{l}\text { - Punition par la loi } \\
\text { (en justice) }\end{array}$ \\
$\begin{array}{l}\text { Rencontre avec un } \\
\text { intervenant }\end{array}$ & $\begin{array}{l}\text { Plan de } \\
\text { Punition civile } \\
\text { (p. ex., travaux } \\
\text { communautaires) }\end{array}$ \\
$\begin{array}{l}\text { Sanction créative } \\
\text { (p. ex., affiches } \\
\text { anticyberintimidation) }\end{array}$ & $\begin{array}{l}\text { comportement } \\
\text { Éducation à la } \\
\text { citoyenneté }\end{array}$ & - Suspension \\
& - Expulsion \\
& Extracurriculaire & \\
& - Retenue & \\
\hline
\end{tabular}

Remarque : Traduction libre d'Hinduja et Patchin (2009), p. 164.

Le modèle écologique de Bronfenbrenner (1979) suggère que plusieurs influences agissent sur le développement humain et viennent de l'environnement immédiat de l'enfant, comme sa famille, son école et ses pairs (microsystème), des interrelations entre ces composantes (mésosystème), et de l'ensemble des environnements plus éloignés, comme le milieu de travail des parents (exosystème) et les valeurs, idéaux culturels et caractéristiques socioéconomiques - taux de chômage, situation politique, etc. - de la communauté (macrosystème). Il s'agit là de facteurs issus des interactions existantes dans l'environnement de l'individu et à partir desquelles il se développe et apprend à se comporter. Depuis sa création, le modèle écologique de Bronfenbrenner (1979) s'est vu enrichir par la littérature scientifique par l'ajout d'une 
dimension biologique qui souligne l'existence de prédispositions génétiques agissant sur le développement comportemental de l'individu (ontosystème) (Bronfenbrenner \& Ceci, 1994; Dishion \& Stormshak, 2007), et d'une dimension temporelle qui reconnaît l'influence de l'évolution sociale et génétique sur les comportements d'un individu (chronosystème) (Belsky, 1995; Bronfenbrenner, 1995; Bronfenbrenner \& Evans, 2000). Cela suggère que le bagage génétique de celui-ci, l'époque où il vit et les relations et comportements de ses pairs, sa famille, le personnel de son école ou des membres de sa communauté exercent des influences importantes qui détermineront ses comportements. Ces explications justifient l'implication d'autres personnes (parents, pairs, autorités scolaires ou autorités civiles) dans les interventions proposées par le modèle d'Hinduja et Patchin (2009). Par ailleurs, Beaumont (2009, novembre) et Johnson (2010) suggèrent que l'utilisation d'Internet peut s'imposer en tant que nouveau système (le technosystème), puisque les communications en ligne constituent une variable importante dans le développement psychosocial des enfants. L'enseignement et la modélisation des comportements à adopter en ligne sont donc très importants dans une société de plus en plus informatisée.

Même si la plupart des interventions qu'ils suggèrent sont principalement à caractère éducatif, Hinduja et Patchin (2009) recommandent une sanction pénale (p. ex., amende ou travaux communautaires), une suspension ou une expulsion de l'école si l'élève a commis des agressions sérieuses en ligne. À la suite de Piaget (1928/2009) et sa théorie sur la cognition sociale, Bandura (1986) fait valoir que l'autorégulation des comportements s'effectue en fonction des expériences vécues. De fait, il suggère que l'individu apprend de ses expériences, répétant les comportements qui lui ont procuré des satisfactions et évitant de reproduire ceux qui ont eu des conséquences négatives. Dans une telle optique, les punitions (sanctions désagréables) du modèle d'intervention de Hinduja et Patchin (2009) pourraient permettre l'autorégulation des comportements en amenant l'individu à agir de manière à éviter l'expérience négative.

Les limites du modèle d'Hinduja et Patchin (2009) se situent d'abord dans le choix des interventions, qui tiennent compte de la gravité du geste posé, mais pas des motivations de l'élève. Par exemple, ce pourrait être important qu'un élève qui fait des menaces de mort (gravité sévère) reçoive aussi un enseignement en habiletés sociales pour qu'il apprenne à entrer en relation avec les autres d'une manière acceptable (Andreou, 2006; Björkqvist, Österman, \& Kaukiainen, 2000). De plus, si 
on considère les écrits de certains chercheurs (Ang \& Goh, 2010; Joliffe \& Farrington, 2006; Malti, Perren, \& Buchmann, 2009), tous les niveaux de gravité des agressions devraient s'accompagner d'un enseignement en empathie (se mettre dans la peau de l'autre pour comprendre ses émotions). Finalement, ce modèle d'intervention ne considère pas la gravité de la répétition ou de la récidive en situation d'agression mineure. Après les sanctions créatives, les rencontres et les discussions, des interventions plus sévères, comme des retenues ou une suspension de l'école, pourraient être nécessaires si un comportement d'agression mineure perdurait. Hinduja et Patchin (2010) notent d'ailleurs le lien entre la persistance des agressions et les idées et comportements suicidaires des victimes, ce qui permet de souligner à quel point le maintien d'un comportement de cyberintimidation qu'on dit mineur est grave.

Le modèle d'intervention d'Hinduja et Patchin (2009) n'a pas encore fait ses preuves en milieu scolaire. Il devra encore faire l'objet d'un examen empirique systématique, mais pour l'instant, la présente étude permet de le confronter aux interventions des enseignants en réaction aux comportements de cyberintimidation de leurs élèves. Plus précisément, cette étude vise à 1) décrire les types d'intervention utilisés par les enseignants auprès des élèves qui font de la cyberintimidation, 2) identifier les interventions perçues comme étant les plus efficaces, et 3 ) analyser les fondements théoriques ou concepts qui appuient ces interventions.

\section{Méthode}

\section{Participants}

Dix enseignants, soit trois femmes et sept hommes, de deux écoles secondaires et d'une école primaire du Nouveau-Brunswick ont participé à cette étude. Ces enseignants ont à leur charge au moins un groupe d'élèves de $7^{\mathrm{e}}, 8^{\mathrm{e}}$ ou de $9^{\mathrm{e}}$ année, donc âgés de 12 à 15 ans. En moyenne, les participants étaient âgés de 40 ans et comptaient 16 années d'expérience à ces niveaux. L'âge et l'expérience des participants n'ont pas été considérés dans leur sélection, ni dans l'analyse des résultats, étant donné que le problème de la cyberintimidation a connu son expansion dans la dernière décennie seulement et que les enseignants, d'expérience ou non, doivent régler des situations pour lesquelles il existe encore peu d'outils. 


\section{Matériel et procédure}

Dans le cadre d'entrevues semi-dirigées enregistrées, les participants ont été interrogés sur leurs choix d'interventions en réponse aux comportements de cyberintimidation des élèves. Afin de limiter leurs divergences d'opinions concernant la définition de la cyberintimidation, ils ont eu droit à deux exemples de comportements de cyberintimidation pour chacun des trois niveaux de gravité proposés par Hinduja et Patchin (2009), donnant lieu à six mises en situation. Par exemple, une des mises en situation pour un acte de cyberintimidation de gravité mineure était : "On vous rapporte qu'un élève de votre classe traite de noms un autre élève sur un site Internet. Une situation semblable est-elle déjà arrivée? " En cas de réponse affirmative, les enseignants devaient décrire la situation et expliquer comment ils étaient intervenus; en cas de réponse négative, ils devaient expliquer comment ils auraient réagi si la situation s'était présentée. On a aussi demandé aux participants d'expliquer pourquoi ils avaient choisi ou choisiraient un type d'intervention plutôt qu'un autre afin d'analyser sur quelles bases théoriques ou quels concepts s'appuyaient leurs actions. Enfin, les participants ont été invités à partager leurs expériences avec la cyberintimidation, décrire leurs interventions et exprimer leur perception de l'efficacité de ces dernières.

\section{Résultats}

Les enregistrements des propos recueillis ont été retranscrits pour être soumis à une analyse de contenu qui a permis de comparer les interventions (Gauthier, 2008). Les interventions semblables choisies par les enseignants ont été regroupées afin de faire ressortir celles auxquelles ils avaient donné priorité. À chacun des niveaux de gravité des comportements, toute intervention rapportée a été traduite en " unité de sens » et comptabilisée (Leray, 2008; Paillé \& Mucchielli, 2003). Par exemple, si deux enseignants ont dit qu'ils communiqueraient avec les parents de l'agresseur à la situation numéro 1 (à la suite d'un acte de gravité mineure), on a compté deux unités de sens pour cette intervention. Après coup, les données ont fait l'objet d'une analyse nourrie des approches théoriques sur la modification des comportements que les responsables de l'étude avaient dégagés de la littérature. 


\section{Les interventions pratiquées par les enseignants auprès des jeunes qui s'adonnent à la cyberintimidation}

Un premier niveau d'analyse a permis de regrouper les interventions des enseignants dans les catégories suivantes :

1) interventions liées à l'éducation par l'enseignant (p. ex., explication des conséquences de la cyberintimidation);

2) interventions liées à l’implication des parents (p. ex., rencontre avec les parents);

3) interventions liées à l'implication de la direction (p. ex., suspension, expulsion);

4) interventions liées à l'implication d'un policier (éducation, enquête, sanction, prévention);

5) interventions liées à la prévention auprès des autres élèves (discussion) pour éviter que la cyberintimidation ne se reproduise;

6) interventions liées à la modification du comportement impliquant un professionnel (p. ex., apport d'un travailleur social ou d'un spécialiste en modification de comportement);

7) interventions liées à la sanction donnée par l'enseignant (p. ex., retenue après la classe), et

8) interventions visant la réparation des torts causés (p. ex., effacement des traces d'agressions en ligne, excuses).

Le nombre de fois que chaque catégorie d'intervention a été choisie par les participants pour répondre à des agressions mineures, modérées et sévères s'est lui aussi traduit en unités de sens. Par exemple, si un participant proposait d'appeler à la maison pour aviser les parents de l'élève ayant commis un acte de cyberintimidation, on comptait une unité dans la catégorie Interventions liées à l'implication d'un parent.

Quelques-unes des situations hypothétiques proposées s'étaient déjà manifestées aux enseignants interrogés : trois participants avaient vécu une des six situations, quatre en avaient connu deux et un avait été exposé à trois. Par ailleurs, aucun participant n'a mentionné avoir eu à gérer de situation où l'agresseur était inconnu.

Létude n'a révélé aucune différence notable entre les interventions suggérées pour répondre aux situations fictives de cyberintimidation décrites dans les vignettes et les interventions choisies par les enseignants dans la réalité. Par ailleurs, dans plus de $70 \%$ des situations proposées, les participants ont suggéré plusieurs interventions possibles. 
Certaines interventions s'avèrent dominantes pour l'ensemble des situations proposées, avec une importance plus marquée pour certains niveaux de gravité du geste commis. On retrouve au premier rang les interventions liées à l'éducation (29 unités de sens). Les participants ont justifié ce choix en disant que c'est leur rôle d'éduquer les jeunes : «C'est notre mandat. " « On veut que ce soit éducatif tout ça. " « L'utilisation des technologies, c'est une éducation. » Au deuxième rang, à égalité (26 unités de sens), figurent les interventions liées à l'implication d'un parent et celles liées à l'implication de la direction. Le choix de l'implication d'un parent s'est justifié par l'importance du rôle des parents dans la supervision de l'utilisation des technologies disponibles à la maison et dans leur éducation comportementale générale : "Ce sont les parents qui ont le contrôle à la maison. " "Les premiers responsables de l'éducation, de la santé et du bien-être de l'enfant, ce sont les parents, pas l'école. » Les interventions liées à l'implication de la direction se sont justifiées par des sentiments d'impuissance chez les enseignants, surtout en ce qui concerne l'application de sanctions : "Je vais être limité dans l'intervention. " "Comme enseignant, il y a des choses que tu ne peux pas te permettre de faire. " Ces sentiments expliquent aussi la forte représentation ( 21 unités de sens) des interventions liées à l'implication d'un policier, auquel les participants attribuent plusieurs rôles dans l'intervention, soit l'éducation, l'enquête, la sanction et la prévention : «[...] comme démarche, peut-être rencontrer un policier éducateur. " «Je suppose que le policier éducateur pourrait retracer l'information.» "L'intervention des policiers [de la] GRC [...] par rapport à la saisie du téléphone." "Intervenir en faisant venir un policier pour expliquer au groupe jusqu'où ça pourrait aller.» Ces interventions impliquant un policier ont été mentionnées le plus souvent en réaction aux comportements de gravité sévère (12 unités de sens). Finalement, des actions liées à la prévention auprès des autres élèves ont été soulevées à plusieurs reprises (10 unités de sens), car elles permettent de rassurer les élèves, mais aussi de prévenir d'autres situations de cyberintimidation : "Il faut rassurer les autres élèves. " "Il faut informer les enfants sur comment se servir de ça. » " [...] pour qu'ils puissent réagir autrement s'ils l'entendent [la rumeur]. »

\section{La perception de l'efficacité des interventions pour contrer la cyberintimidation}

Six participants sur 10 croient que l'efficacité de l'intervention dépend d'abord de l'élève lui-même, de sa réceptivité aux interventions et de 
son attitude. Toutefois, parmi les interventions jugées les plus efficaces par les enseignants, on retrouve, par ordre d'importance, 1) l'implication des parents, 2) le recours à la loi, 3) l'éducation par la discussion, 4) l'implication de la direction, et 5) l'intervention visant la réparation du tort causé à la victime.

Nommée par six enseignants, l'attitude de réceptivité de l'élève s'impose comme le facteur déterminant d'une intervention efficace. "On a des bullies à l'école, puis on dirait qu'à chaque année, même si tu leur donnes de la formation, même si tu les sensibilises à ça, même si tu jases avec eux autres, l'année d'après ils sont encore des bullies, même la semaine d'après. » « On le fait par principe, pour essayer d'aider d'autres élèves, mais est-ce que c'est efficace? Pas nécessairement. [L]a personne qui était consciente, même si tu l'amènes en période de réflexion, ne care pas ». Six enseignants considèrent toutefois que l'implication d'un policier, autant comme éducateur qu'agent de police, peut s'avérer efficace pour faire cesser les comportements inacceptables, puisqu'en présence policière, les élèves prennent la situation plus au sérieux : "C'est lorsque c'est rendu plus loin, par exemple, quand les policiers sont rendus dans le dossier, que là, on dirait qu'ils commencent à réaliser, mais avant ça, c'est juste une joke plate. » Six participants sont aussi d'avis qu'avec la collaboration et un suivi des parents, les interventions ont une meilleure chance de réussir : "On a généralement un très bon succès quand on travaille en collaboration avec les parents. » Un participant convaincu des bienfaits pour l'élève d'un travail de conscientisation pense toutefois que les interventions contre la cyberintimidation peuvent difficilement être totalement efficaces, car les dommages suivent trop souvent la victime après que les comportements d'agression aient cessé. Selon lui, le problème ne sera pas réglé même après une intervention qui semble avoir fonctionné : « Ça peut peut-être le régler au niveau de la personne qui intimide, qu'il arrête, mais ça ne le règlera pas au niveau de ce qui a déjà été fait. »Ce même participant a proposé, parmi ses interventions, de demander à l'agresseur d'essayer de réparer ses torts en ligne.

\section{Quelques caractéristiques du choix des interventions pour contrer la cyberintimidation}

La littérature scientifique nous permet d'analyser les bases sur lesquelles les enseignants s'appuient pour choisir leurs interventions. Il faut cependant souligner qu'aucun participant n'a dit s'appuyer sur une théorie en particulier en réponse à la question : "Pourquoi avezvous choisi cette intervention? » À la lumière des propos recueillis, on 
constate que leurs réponses relèvent davantage de leurs expériences d'intervention dans différents contextes où il s'est agi d'atténuer un comportement indésirable : «[...] ces choses-là que je t'ai répondues, c'est par rapport à mon expérience. » Par ailleurs, six enseignants ont dit se sentir très limités dans leur capacité d'intervenir. De plus, 9 répondants sur 10 s'estiment insuffisamment équipés ou informés pour composer adéquatement avec la cyberintimidation : « Je trouve qu'on n'est pas très très outillés, pas beaucoup au courant de ce qu'on peut faire, de comment on peut s'y prendre. " Tous les enseignants interrogés ont d'ailleurs mentionné qu'ils n'interviendraient pas seuls, mais demanderaient la collaboration d'autres personnes (direction de l'école, parents, policier, travailleur social ou spécialiste en modification de comportement), indépendamment du niveau de gravité des gestes de cyberintimidation posés.

\section{Analyse des interventions soulevées en fonction des bases théoriques dégagées de la littérature}

La littérature dégage quatre approches pouvant influencer les comportements d'agression : le développement de l'empathie (Ang \& Goh, 2010), le développement des habiletés sociales (Andreou, 2006; Björkqvist et al., 2000), l'autorégulation comportementale par la sanction (Bandura, 1986; Piaget, 1928/2009) et l'influence des facteurs environnementaux (Bronfenbrenner, 1979). Afin de voir dans quelle mesure les interventions des enseignants en réponse à la cyberintimidation correspondent à des pratiques efficaces pour remédier à un comportement non désiré, elles ont été mises en perspective avec les quatre approches nommées ci-dessus. Le développement de l'empathie (réflexion par rapport aux torts causés à la victime), d'abord, pourrait s'appliquer aux interventions liées à l'éducation par l'enseignant, à la réparation des torts et à la prévention auprès des autres élèves. Le développement des habiletés sociales (compréhension des dynamiques sociales, habiletés de communication et de gestion de conflits) pourrait valoir pour les interventions liées à l'éducation par l'enseignant, à l'implication d'un policier (éducation et prévention), à la réparation des torts, à la modification d'un comportement impliquant un professionnel et à la prévention auprès des autres élèves. L'autorégulation comportementale (par la sanction) pourrait concerner les interventions associées à l'implication de la direction (expulsion, suspension ou autre punition), à l'implication d'un policier (sanction) et aux mesures disciplinaires imposées par l'enseignant. Finalement, l'influence des 
facteurs environnementaux sur les comportements d'agression pourrait correspondre aux interventions liées à l'implication des parents, de la direction ou d'un policier, et à la prévention auprès des autres élèves. On peut établir d'autres liens entre les interventions soulevées par les participants et ces quatre approches selon la nature des démarches entreprises par les différents intervenants. Par exemple, un directeur d'école pourrait opter pour une intervention punitive, alors qu'un autre ferait davantage d'éducation au sujet des comportements empathiques (voir Tableau 2).

\section{Tableau 2 - Liens possibles entre les interventions proposées par les participants et quatre approches théoriques}

\begin{tabular}{|l|c|c|c|c|}
\hline & $\begin{array}{c}\text { Enseignement } \\
\text { des } \\
\text { habiletés } \\
\text { sociales }\end{array}$ & $\begin{array}{c}\text { Enseignement } \\
\text { de } \\
\text { comportements } \\
\text { empathiques }\end{array}$ & $\begin{array}{c}\text { Influences } \\
\text { de } \\
\text { l'environnement }\end{array}$ & $\begin{array}{c}\text { Auto- } \\
\text { régulation } \\
\text { comporte- } \\
\text { mentale }\end{array}$ \\
\hline $\begin{array}{l}\text { 1. Interventions liées } \\
\text { à l'éducation par } \\
\text { l'enseignant }\end{array}$ & $\mathrm{X}$ & $\mathrm{X}$ & $\mathrm{X}$ & \\
\hline $\begin{array}{l}\text { 2. Interventions liées à } \\
\text { l'implication des parents }\end{array}$ & $\mathrm{X}$ & $\mathrm{X}$ & $\mathrm{X}$ \\
\hline $\begin{array}{l}\text { 3. Interventions liées } \\
\text { à l'implication de la } \\
\text { direction }\end{array}$ & $\mathrm{X}$ & $\mathrm{X}$ & $\mathrm{X}$ & $\mathrm{X}$ \\
\hline $\begin{array}{l}\text { 4. Interventions liées à } \\
\text { l'implication d'un policier }\end{array}$ & $\mathrm{X}$ & $\mathrm{X}$ & \\
\hline $\begin{array}{l}\text { 5. Interventions liées à la } \\
\text { prévention auprès des } \\
\text { autres élèves }\end{array}$ & $\mathrm{X}$ & $\mathrm{X}$ & $\mathrm{X}$ \\
\hline $\begin{array}{l}\text { 6. Interventions liées } \\
\text { à la modification } \\
\text { d'un comportement } \\
\text { impliquant un } \\
\text { professionnel travailleur } \\
\text { social, spécialiste) }\end{array}$ & & & & \\
\hline $\begin{array}{l}\text { 7. Interventions liées à } \\
\text { la sanction donnée par } \\
\text { l'enseignant }\end{array}$ & & & & \\
\hline $\begin{array}{l}\text { 8. Interventions visant } \\
\text { la réparation des torts } \\
\text { causés }\end{array}$ & $\mathrm{X}$ & & \\
\hline Remarque:ce tableau n'epuis & & & \\
\hline
\end{tabular}

Remarque : ce tableau n'épuise pas la liste des liens possibles.

À la suite de cette analyse, on peut croire que les enseignants interrogés ne négligent aucune de ces quatre importantes approches lorsqu'ils interviennent auprès des élèves qui cyberintimident leurs pairs, car leurs principaux modes d'intervention (éducation par 
l'enseignant, implication des parents, de la direction ou d'un policier, prévention auprès des autres élèves) rejoignent chacune d'elles à différents degrés. Rappelons toutefois que ce sont leurs expériences qui ont conduit les participants vers ces choix d'intervention, et non leurs connaissances théoriques.

\section{Discussion}

Après avoir associé les interventions rapportées par les participants à quatre approches relativement à leur potentiel d'efficacité, nous les avons mises en perspective avec le modèle théorique d'Hinduja et Patchin (2009) pour voir dans quelle mesure cet outil pourrait répondre aux besoins et aux attentes des enseignants. La suite du texte aborde aussi les interventions jugées efficaces par les enseignants et les enjeux liés à la formation de ceux-ci.

\section{Les interventions des enseignants par rapport au modèle d'Hinduja et Patchin}

Le modèle théorique d'Hinduja et Patchin (2009) propose des interventions en réponse à trois niveaux de gravité de comportements de cyberintimidation, et les interventions des enseignants interrogés s'y retrouvent (p. ex., l'éducation, l'implication de la direction ou des parents, le recours à la loi, les activités créatives, les travaux communautaires, la punition). Toutefois, d'autres types d'intervention faits par les enseignants (p. ex., la réparation, la discussion préventive avec le groupe) n'y figurent pas. En effet, les enseignants rapportent souvent des interventions liées à l'éducation pour chacun des niveaux de gravité, alors que le modèle d'Hinduja et Patchin propose ce genre d'intervention pour les comportements de gravité mineure ou modérée exclusivement.

Du point de vue des actions qui recourent à l'application de la loi, des similitudes s'observent aussi entre les réponses des participants et le modèle d'Hinduja et Patchin en ce qui concerne la rencontre des élèves avec un policier relativement à des comportements de gravité sévère. Certains enseignants ont aussi mentionné ce recours pour répondre à des agressions mineures et modérées. Les mêmes interventions sont d'ailleurs les plus souvent mentionnées à chacun des niveaux d'agression, ce qui laisse croire que plusieurs enseignants n'interviennent pas systématiquement en s'inspirant de la gravité du comportement. 
On se rappellera que la sévérité des interventions proposées par le continuum d'Hinduja et Patchin (2009) dépend de la gravité des actes commis. Dans la présente étude, six des 10 enseignants interrogés ont mentionné que la sévérité des interventions qu'ils choisissaient dépendait aussi des antécédents comportementaux de l'élève et de sa réceptivité à une première intervention.

Le modèle théorique d'Hinduja et Patchin accorde une attention particulière aux plans de modification de comportement (plans d'intervention) et aux sanctions créatives (p. ex., affiches anticyberintimidation), alors qu'aucun enseignant interrogé n'a dit proposer ces types d'intervention. Certains participants ont d'ailleurs mentionné préférer les interventions éducatives misant sur la discussion et le travail de réflexion. Puisque plusieurs d'entre eux se sentent mal outillés pour réagir à ce nouveau type de violence et qu'ils veulent jouer un rôle d'éducation, il serait pertinent de les guider vers un modèle d'intervention susceptible de servir auprès des élèves qui pratiquent la cyberintimidation.

\section{Les éléments considérés par les enseignants lorsqu'ils choisissent leurs interventions}

Dans sa deuxième phase, l'analyse a relié les interventions des enseignants aux quatre approches théoriques sur la modification d'un comportement que nous avons dégagées de la littérature : développement de l'empathie, développement des habiletés sociales, autorégulation comportementale (par la sanction) et facteurs environnementaux (implication des parents ou d'autres personnes de la communauté). Cela a permis de constater que les interventions privilégiées par les enseignants sont potentiellement efficaces selon la littérature scientifique sur la question. Les participants n'ont toutefois pas fait référence à ces approches pour expliquer leurs choix d'interventions, mais bien à leur responsabilité professionnelle et aux limites de leur rôle d'éducateur. Il faut envisager que leurs choix correspondent à des interventions aussi utilisées pour répondre à d'autres types de comportements violents.

À part la gravité et la persistance des comportements, les motifs et les raisons qui influencent les réactions des enseignants envers des comportements de cyberintimidation reposent souvent sur la responsabilité d'éduquer qu'ils sentent avoir et leurs capacités limitées à intervenir, ce qui les pousse à faire appel à d'autres intervenants. Les 
enseignants interrogés considèrent d'ailleurs que les parents ont un rôle de premier plan dans l'intervention, puisque ce sont eux qui contrôlent l'utilisation des technologies à la maison et que c'est à eux que revient le rôle de supervision. Sans leur renvoyer la balle, les enseignants souhaitent pouvoir collaborer avec les parents. Selon les participants, les parents ont plus de pouvoir que les enseignants relativement à l'utilisation supervisée des technologies.

\section{Un nouveau modèle d'intervention}

À la lumière des résultats obtenus dans le cadre de cette recherche et des approches théoriques étudiées, nous avons créé, de manière post hoc, un modèle en quatre étapes pour intervenir auprès des élèves qui pratiquent la cyberintimidation, incluant 1) la prévention, 2) l'éducation, 3) la sanction ou les conséquences, et 4) le suivi (voir Figure 1). Ce nouveau modèle ne répond pas à un objectif de la présente étude, mais sert plutôt à combler le besoin de pistes d'intervention souligné par les enseignants interrogés.

Plusieurs enseignants ont souligné l'importance de la prévention, la première étape. Celle-ci est dictée par la nécessité d'éduquer les jeunes à la cyberconduite, en ce sens qu'elle permettra de décourager les comportements de cyberintimidation avant qu'ils ne se produisent. $\mathrm{Si}$ un geste de cyberintimidation survient malgré les activités de prévention, l'étape suivante suggère de faire de l'éducation auprès de l'élève agresseur par le biais d'interventions personnalisées (réparation des torts causés, développement de l'empathie, enseignement des habiletés sociales, implication des parents et de la direction). Nous considérons également important de conserver la notion de gravité du geste posé (Hinduja \& Patchin, 2009) dans ce nouveau modèle, puisqu'un acte de cyberintimidation, même isolé, peut causer des dommages considérables à un individu et que certains comportements sont punissables par la loi. La question de la gravité du geste devrait donc peser dans la balance pour décider s'il faut ajouter une sanction aux autres interventions. Si les interventions éducatives personnalisées ne suffisent pas et que l'agresseur récidive, même par un geste de gravité mineure, une action éducative en plus d'une sanction/conséquence, pourrait alors être nécessaire pour dissuader l'élève pour de bon, tout en l'aidant à comprendre la portée de ses actes (retenues, suspension, punition civile, punition légale). De façon générale, toute intervention (réparation des torts causés, plan de modification du comportement, implication des 


\section{Figure 1 - Un modèle d'intervention pour contrer la cyber- intimidation (Prévention-Éducation-Sanctions-Suivi [PESS])}

\begin{tabular}{|c|c|c|}
\hline \multicolumn{3}{|c|}{ ÉTAPE 1 : PRÉVENTION POUR TOUS } \\
\hline \multicolumn{3}{|c|}{$\begin{array}{l}\text { - Activités visant le développement de l'empathie : projets éducatifs, recherches, discussion. } \\
\text { - Activités visant le développement des habiletés sociales : communication, } \\
\text { gestion des émotions, et des conflits, respect des différences. } \\
\text { - Activités visant le développement du civisme : comportements appropriés en ligne, } \\
\text { conséquences civiles et légales. }\end{array}$} \\
\hline \multicolumn{3}{|c|}{ ÉTAPE 2 : ÉDUCATION ET RÉÉDUCATION } \\
\hline $\begin{array}{l}\text { Agressions mineures } \\
\text { et première offense: } \\
\text { interventions éducatives } \\
\text { initiées par l'enseignant. }\end{array}$ & $\begin{array}{l}\text { Agressions modérées (ou } \\
\text { récidive): interventions } \\
\text { éducatives/rééducatives initiées } \\
\text { par l'enseignant et impliquant } \\
\text { d'autres professionnels. }\end{array}$ & $\begin{array}{l}\text { Agressions sérieuses (ou } \\
\text { multiples récidives): } \\
\text { interventions éducatives/ } \\
\text { rééducatives initiées par } \\
\text { l'enseignant et impliquant } \\
\text { d'autres professionnels. } \\
\end{array}$ \\
\hline $\begin{array}{l}\text { - Visant le développement } \\
\text { des habiletés sociales et } \\
\text { citoyennes; } \\
\text { - Implication de la direction; } \\
\text { - Implication des parents; } \\
\text { - Implication d'un policier } \\
\text { éducateur. }\end{array}$ & $\begin{array}{l}\text { - Plan d'intervention (P. I.) } \\
\text { incluant le développement de } \\
\text { l'empathie et des habiletés } \\
\text { sociales et citoyennes; } \\
\text { - Implication des parents; } \\
\text { - Implication de la direction; } \\
\text { - Implication d'un policier } \\
\text { éducateur. }\end{array}$ & $\begin{array}{l}\text { - Plan d'intervention (P. I.) } \\
\text { incluant le développement } \\
\text { de l'empathie, des habiletés } \\
\text { sociales et citoyennes, } \\
\text { et un suivi individuel } \\
\text { (professionnel); } \\
\text { - Implication des parents; } \\
\text { - Implication de la direction; } \\
\text { - Implication d'un policier } \\
\text { éducateur. }\end{array}$ \\
\hline \multicolumn{3}{|c|}{ ÉTAPE 3 : SANCTIONS/CONSÉQUENCES } \\
\hline $\begin{array}{l}\text { - Sanctions/conséquences } \\
\text { créatives (p. ex., affiches } \\
\text { anticyberintimidation); } \\
\text { - Réparation des torts } \\
\text { causés (p. ex., excuses à la } \\
\text { victime et redressement de } \\
\text { sa réputation, etc.). }\end{array}$ & $\begin{array}{l}\text { - Sanctions/conséquences } \\
\text { créatives (p. ex., affiches } \\
\text { anticyberintimidation); } \\
\text { - Réparation des torts causés } \\
\text { (p. ex., excuses à la victime } \\
\text { et redressement de sa } \\
\text { réputation); } \\
\text { - Suspension à l'interne avec } \\
\text { activités de réflexion sur l'acte } \\
\text { commis; } \\
\text { - Conséquences légales : } \\
\text { signalement aux policiers. }\end{array}$ & $\begin{array}{l}\text { - Réparation des torts causés } \\
\text { (p. ex., excuses à la victime } \\
\text { et redressement de sa } \\
\text { réputation); } \\
\text { - Suspension à l'externe avec } \\
\text { activités de réflexion sur } \\
\text { l'acte commis et retour avec } \\
\text { les parents; } \\
\text { - Conséquences légales : } \\
\text { signalement aux policiers. }\end{array}$ \\
\hline \multicolumn{3}{|l|}{ ÉTAPE 4 : SUIVI } \\
\hline $\begin{array}{l}\text { Agressions mineures } \\
\text { et première offense: } \\
\text { interventions éducatives } \\
\text { initiées par l'enseignant. }\end{array}$ & $\begin{array}{l}\text { Agressions modérées (ou } \\
\text { récidive): interventions } \\
\text { éducatives/rééducatives initiées } \\
\text { par l'enseignant et impliquant } \\
\text { d'autres professionnels. }\end{array}$ & $\begin{array}{l}\text { Agressions sérieuses (ou } \\
\text { multiples récidives): } \\
\text { interventions éducatives/ } \\
\text { rééducatives initiées par } \\
\text { l'enseignant et impliquant } \\
\text { d'autres professionnels. }\end{array}$ \\
\hline $\begin{array}{l}\text { - Vérification auprès de la } \\
\text { victime et des témoins } \\
\text { (sécurité de la victime } \\
\text { et comportements de } \\
\text { l'élève qui a commis la } \\
\text { cyberagression). }\end{array}$ & $\begin{array}{l}\text { - Vérification auprès de la } \\
\text { victime et des témoins } \\
\text { (sécurité de la victime et } \\
\text { comportements de l'élève qui a } \\
\text { commis la cyberagression); } \\
\text { - Rencontre de suivi au P. I. }\end{array}$ & $\begin{array}{l}\text { - Vérification auprès de la } \\
\text { victime et des témoins } \\
\text { (sécurité de la victime } \\
\text { et comportements de } \\
\text { l'élève qui a commis la } \\
\text { cyberagression); } \\
\text { - Rencontre de suivi au P. I. }\end{array}$ \\
\hline
\end{tabular}


parents et de la direction, activités centrées sur le développement de l'empathie) devrait faire l'objet d'un suivi de manière à s'assurer de son efficacité dans la durée.

Ce nouveau modèle d'intervention, qui allie à la fois des éléments proposés par Hinduja et Patchin (2009) (l'utilisation de sanctions créatives et la considération de la gravité des gestes commis dans le choix des interventions) et d'autres issus de la présente recherche (la gravité de la répétition ou de la récidive des agressions (voir Tableau 3) et l'importance de l'enseignement aux habiletés sociales et aux comportements empathiques), pourrait s'avérer fort utile en milieu scolaire. Un tel modèle peut guider les enseignants relativement aux mesures à prendre selon l'ampleur des gestes de cyberintimidation posés, mais aussi leur permettre d'articuler leur programme d'intervention en fonction d'étapes potentiellement efficaces. D'ailleurs, la croyance voulant que l'efficacité de l'intervention dépende de la réceptivité des élèves, alors que c'est à l'adulte d'agir en éducateur dans ce type de situation, traduit un certain sentiment d'impuissance des enseignants envers leur réelle capacité à intervenir utilement, et leur manque de formation en matière de cyberintimidation y est peut-être pour quelque chose.

Il est possible de faire un pont entre les interventions que nous proposons et la littérature scientifique à ce sujet. Tout d'abord, dans notre modèle d'intervention, les activités qui visent le développement de l'empathie (Ang \& Goh, 2010) et l'enseignement des habiletés sociales (Andreou, 2006; Björkqvist et al., 2000) sont essentielles, et donc présentes dès l'étape de la prévention. Pour favoriser le succès de l'intervention en prévenant la récidive et veillant à ce que les victimes se sentent désormais en sécurité, qu'il y ait eu ou non une sanction/conséquence, nous avons ajouté l'étape du suivi. L'implication des parents, jugée importante par les enseignants, mais aussi dans la littérature (Bhat, 2008; Hinduja et Patchin, 2009), occupe une place importante dans notre modèle.

Des limites s'imposent à l'utilisation de ce modèle, puisqu'il permet seulement de traiter les situations de cyberintimidation où l'agresseur est connu. Dans certains cas, avant d'entreprendre l'étape 2 (éducation et rééducation) et l'étape 3 (sanction/conséquences), une étape intermédiaire d'enquête pourrait s'avérer nécessaire pour démystifier le problème et connaître à la fois les personnes impliquées et les motifs des agressions. 


\section{Des enjeux importants : le sentiment d'inefficacité des enseignants et le manque de connaissances théoriques}

Plusieurs enseignants ont souligné qu'ils se sentent peu efficaces dans leurs interventions contre la cyberintimidation et que l'attitude non réceptive d'un élève pourrait compromettre la réussite d'une intervention et peut-être les décourager à persévérer auprès d'eux. Ce sentiment d'inefficacité des enseignants pourrait prendre sa source dans leur manque de formation pour composer avec les comportements violents, notamment ceux de cyberintimidation de leurs élèves (Royer, 2005). L'offre d'incitatifs à travailler en collaboration avec des collègues et celle de formations spécialisées sont des mesures qui pourraient contribuer à augmenter la confiance des enseignants de pouvoir répondre convenablement aux comportements de cyberintimidation (Bandura, 2003; Bandura, Barbaranelli, Caprara, \& Pastorelli, 1996; Sela-Shayovits, 2009).

Rappelons que l'intervention la plus populaire contre l'ensemble des situations de cyberintimidation présentées aux participants de cette étude a été l'éducation de l'élève aux conséquences de la cyberintimidation par la discussion. Pour les aider à intervenir par l'éducation, il pourrait donc être pertinent d'offrir aux enseignants des formations sur le phénomène et ses conséquences pour les victimes, la résolution de conflits ou la communication. Hinduja et Patchin (2009) suggèrent d'ailleurs d'utiliser des interventions éducatives comme des projets ou des recherches sur les conséquences de la cyberintimidation, ou de créer des plans de modification de comportement pour les élèves qui agressent leurs pairs en ligne. Paul, Smith et Blumbert (2010) suggèrent aussi que la création de groupes de discussion composés d'élèves ayant pour mission de trouver des solutions aux situations de cyberintimidation et de faire de la sensibilisation constitue une pratique efficace pour contrer le problème. Les approches en justice réparatrice (Gromet \& Darley, 2009) que la formation initiale ou continue des enseignants pourrait contenir aideraient l'agresseur à réparer ses torts auprès de la victime, à se conscientiser par rapport aux conséquences et à se sentir comme une meilleure personne. De tels moyens d'intervention, s'ils étaient proposés aux enseignants lors de leur formation initiale ou continue, pourraient contribuer à améliorer leur sentiment d'efficacité comme intervenants (Bandura, 2003; Bandura et al., 1996). Cela leur permettrait également de remplir un rôle d'éducateur auprès des jeunes qui pratiquent la cyberintimidation et de soutien auprès des victimes. 


\section{Tableau 3 - Gravité des gestes posés}

\begin{tabular}{|l|l|}
\hline $\begin{array}{l}\text { Agressions mineures } \\
\text { (atteintes à la dignité) }\end{array}$ & $\begin{array}{l}\text { Agressions modérées (atteinte à la dignité } \\
\text { et l'intégrité morale) à sérieuses (atteinte à } \\
\text { la dignité et à l'intégrité morale et physique) }\end{array}$ \\
\hline - Taquiner & - Nuire à la réputation \\
- Ignorer & - Répandre des rumeurs \\
- Traiter de noms & - Afficher des photos sans permission \\
- Se moquer & - Créer des vidéos intimidantes \\
- Ridiculiser, rabaisser & - Menacer, intimider \\
& - Menacer d'agression physique ou de mort \\
& - Harceler (traquer) \\
\hline
\end{tabular}

\section{Conclusion}

Jusqu'à ce jour, peu de recherches se sont intéressées à la nature des pratiques éducatives mises en place à l'école pour contrer la cyberintimidation entre les jeunes. La présente recherche a permis de recueillir les interventions pratiquées par des enseignants lorsqu'un de leurs élèves a des comportements de cyberintimidation de gravité mineure, modérée ou sévère (Hinduja \& Patchin, 2009). Les conclusions les plus intéressantes à tirer de cette étude sont que les enseignants choisissent davantage leurs interventions en fonction des antécédents comportementaux de l'élève que de la gravité du comportement de cyberintimidation. De plus, ils attribuent les chances de réussite des interventions au niveau de coopération et d'ouverture des élèves plus qu'à la qualité de leurs propres actions. Les résultats suggèrent également que les enseignants préfêrent jouer un rôle éducatif dans l'intervention au lieu de recourir à la sanction ou d'en appeler à la loi. Plusieurs considèrent aussi manquer de moyens pour faire face au phénomène de la cyberintimidation et croient que leur efficacité dans l'action pourrait être accrue si une formation initiale les préparait mieux à intervenir dans ce type de situation. À cet effet, nous avons proposé un nouveau modèle d'intervention considérant à la fois le niveau de collaboration de l'élève et la gravité des gestes d'agression pour mieux répondre aux besoins des enseignants, tout en tenant compte des interventions potentiellement efficaces soulevées par la littérature. Le modèle que nous avançons demeure théorique; il est donc difficile d'en évaluer l'efficacité réelle. Il sera pertinent, dans une étude ultérieure, de vérifier sa capacité d'enrayer les comportements de cyberintimidation chez les jeunes et de répondre aux besoins des enseignants. 
Menée auprès d'un très petit échantillon $(n=10)$ de participants, cette étude ne permet pas de généraliser les résultats à l'ensemble des enseignants. Elle contribue néanmoins à améliorer les connaissances dans ce domaine de recherche encore très jeune, donnant quelques lignes directrices pour orienter les interventions.

\section{Références}

Andreou, E. (2006) Social preference, perceived popularity and social intelligence: Relations to overt and relational aggression. School Psychology International, 27, 339-351. doi:10.1177/0143034306067287

Ang, R. P., \& Goh, D. H. (2010). Cyberbullying among adolescents: The role of affective and cognitive empathy, and gender. Child Psychiatry \& Human Development, 41, 387-397. doi:10.1007/s10578-010-0176

Bandura, A. (2003). Auto-efficacité : le sentiment d'efficacité personnelle. Bruxelles, Belgique : De Boeck.

Bandura, A. (1986). Social foundations of thought and action: A social cognitive theory. Englewood Cliffs, NJ : Prentice-Hall.

Bandura, A., Barbaranelli, C., Caprara, G. V., \& Pastorelli, C. (1996). Multifaceted impact of self-efficacy beliefs on academic fonctionning. Child Development, 67(3), 1206-1222. doi:10.1111/j.1467-8624.1996. tb01791.x

Beaumont, C. (2009, novembre). Des actions collectives pour influencer le développement social des jeunes. Conférence d'ouverture prononcée dans le cadre du Forum provincial organisé par le ministère de l'Éducation, du Loisir et du Sport sur la violence à l'école. Québec, Canada.

Belsky, J. (1995) Expanding the ecology of human development: An evolutionary perspective. Dans P. Moen, G. Elder jr et K. Luscher (dir.), Examining lives in context: Perspectives on the ecology of human development (pp. 545-561). Récupéré de http://psycnet.apa.org.ezproxy. bibl.ulaval.ca/books/10176/015. doi:10.1037/10176-015

Bhat, C. S. (2008). Cyber bullying: Overview and strategies for school counsellors, guidance officers, and all school personnel. Australian Journal of Guidance \& Counselling, 8(1), 53-56. doi:10.1375/ajgc.18.1.53

Björkqvist, K., Österman, K., \& Kaukiainen, A. (2000). Social intelligence Empathy = aggression? Aggression and Violent Behavior, 5(2), 191-200. doi:10.1016/S1359-1789(98)00029-9

Bronfenbrenner, U. (1995). Developmental ecology through space and time: A future perspective. Dans P. Moen, G. Elder jr et K. Luscher (dir.), Examining lives in context: Perspectives on the ecology of human development (pp. 619-647). Récupéré de http://psycnet.apa.org.ezproxy. bibl.ulaval.ca/books/10176/018. doi:10.1037/10176-018

Bronfenbrenner, U. (1979). The ecology of human development: Experiments by nature and design. Cambridge, MA : Harvard University Press. 
Bronfenbrenner, U., \& Ceci, S. J. (1994). Nature-nurture reconceptualized in developmental perspective: A bioecological theory. Psychological Review, 101(4), 568-586. doi:10.1037/0033-295X.101.4.568

Bronfenbrenner, U., \& Evans, G. W. (2000). Developmental science in the $21^{\text {st }}$ century: Emerging questions, theoretical models, research designs and empirical findings. Social Development, 1(9), 116-125. doi:10.1111/14679507.00114

Calvete, E., Orue, I., Villardón, A. E., \& Padilla, P. (2010). Cyberbullying in adolescents: Modalities and aggressors' profile. Computers in Human Behavior, 26, 1128-1135. doi:10.1016/j.chb.2010.03.017

Cassidy, W., Jackson, M., \& Brown, K. N. (2009). Sticks and stones can break my bones, but how can pixels hurt me? School Psychology International, 30(4), 383-402. doi:10.1177/0143034309106948

Conway, K. P., \& McCord, J. (2002). A longitudinal examination of the relation between co-offenders with violent accomplices and violent crime. Aggressive Behavior, 28(2), 97-108. doi:10.1002/ab.90011

Dehue, F., Bolman, C., \& Völlink, T. (2008). Cyberbullying: Yougsters' experience and parental perception. CyberPsychology \& Behavior, 11(2), 217-222. doi:10.1089/cpb.2007.0008

Dishion, T. J., \& Stormshak, E. A. (2007). The ecology development and change. Dans Intervening children's lives: An ecological family-centered approach to mental health care (pp. 15-29). Washington, DC : American Psychology Association. doi:10.1037/11485-002

Erdur-Baker, Ö. (2010). Cyberbullying and its correlation to traditional bullying, gender and frequent and risky usage of internet-mediated communication tools. New Media \& Society, 12(1), 109-125. doi:10.1177/1461444809341260

Gauthier, B. (2008). Recherche sociale : de la problématique à la collecte de données ( $5^{\mathrm{e}}$ éd.). Montréal, Canada : Presses de l'Université du Québec.

Gromet, D. M., \& Darley, J. M. (2009). Punishment and beyond: Achieving justice through the satisfaction of multiple goals. Law and Society Association, 43(1), 1-37. doi:10.1111/j.1540-5893.2009.00365.x

Hinduja, S., \& Patchin, J. W. (2010). Bullying, cyberbullying and suicide. Archives of Suicide Research, 14, 206-621. doi:10.1080/13811118.2010. 494133

Hinduja, S., \& Patchin, J. W. (2009). Bullying beyond the schoolyard. Thousand Oakes, CA : Corwin Press.

Johnson, G. M. (2010). Internet use and child development: Validation of the ecology techno-subsystem. Educational Technology \& Society, 13(1), 176-185.

Joliffe, D., \& Farrington, D. P. (2006). Examining the relationship between low empathy and bullying. Aggressive Behavior, 32, 540-550. doi:10.1002/ ab.20154 
Jones, S. E., Manstead, A. S. R., \& Livingstone, A. G. (2011). Ganging up or sticking together? Group processes and children's responses to text-message bullying. British Journal of Psychology, 102, 71-96. doi:10.1348/000712610x502826

Klomek, A. B., Sourander, A., \& Gould, M. (2010). The association of suicide and bullying in childhood to young adulthood: A review of cross-sectional and longitudinal research finding. Revue canadienne de psychiatrie, 55(5), 282-288.

König, A., Gollwitzer, M., \& Steffgen, G. (2010). Cyberbullying as an act of revenge? Australian Journal of Guidance \& Couselling, 20(2), 210-224. doi:10.1375/ajgc.20.2.210

Leray, C. (2008). L’analyse de contenu de la théorie à la pratique. Montréal, Canada : Presses de l'Université du Québec.

Malti, T., Perren, S., \& Buchmann, M. (2009). Children's peer victimization, empathy, and emotional symptoms. Child Psychiatry \& Human Development, 41, 98-113. doi:10.1007/s10578-009-0155-8

O’Brennan, L. M., Bradshaw, C. P., \& Sawyer, A. L. (2009). Examining developmental differences in the social-emotional problems among frequent bullies, victims, bully/victims. Psychology Schools, 46(2), 100114. doi:10.1002/pits.20357

Paillé, P., \& Mucchielli, A. (2003). L'analyse qualitative en sciences humaines et sociales. Paris, France : Armand Colin.

Paul, S., Smith, P. K., \& Blumbert, H. H. (2010). Adressing cyberbullying in school using the quality circle approach. Australian Journal of Guidance \& Couselling, 20(2), 157-168. doi:10.1375/ajgc.20.2.157

Piaget, J. (2009). Children's understanding of causality. British Journal of Psychology, 100, 207-224. (Article initialement paru en 1928 dans British Journal of Psychology, 18, 276-301.)

Prud'homme, D. (2008). Violences entre enfants : casse-tête pour les parents. Montréal, Canada : Remue-ménage.

Royer, E. (2005). Comme un caméléon sur une jupe écossaise ou Comment enseigner à des jeunes difficiles sans s'épuiser. Québec, Canada : École et comportement.

Sela-Shayovits, R. (2009). Dealing with school violence: The effect of school violence prevention training on teachers' self-efficacy in dealing with violent events. Teaching and Teachers Education, 25, 1061-1066. doi:10.1016/j.tate.2009.04.010

Shariff, S. (2005). Cyber-dilemmas in the new millennium: School obligations to provide student safety in a virtual school environment. McGill Journal of Education, 40(3), 457-477.

Slonje, R., \& Smith, P. K. (2008). Cyberbullying: Another main type of bullying? Scandanavian Journal of Psychology, 49, 147-154. doi:10.1111/ j.1467-9450.2007.00611.x 
Smith, P. K., Mahdavi, J., Carvahlo, M., Fisher, S., Russel, S., \& Tippett, N. (2008). Cyberbullying: Its nature and impact on secondary school pupils. Journal of Child Psychology and Psychiatry, 49(4), 376-385. doi:10.1111/ j.1469-7610.2007.01846.x

Sourander, A., Klomek, A. B., Ikonen, M., Lindroos, J., Luntamo, T., Koskelainen, ...Helenius, H. (2010). Psychosocial risk factors associated with cyberbullying among adolescents: A population based study. Archives of General Psychiatry. 67(7), 720-728. doi:10.1001/ archgenpsychiatry.2010.79

Suzuki, H., \& Lucas, L. R. (2010). Chronic passive exposure to aggression escalates aggressiveness of rat observers. Aggressive Behavior, 36(1), 5466. doi:10.1002/ab.20333

Vandebosch, H., \& van Cleemput, K. (2008). Defining cyberbullying: A qualitative research into the perceptions of youngsters. CyberPsychology \& Behavior, 11(4), 499-50. doi:10.1089/cpb.2007.0042

Walrave, M., \& Heirman, W. (2011). Cyberbullying: Predicting victimization and perpetration. Children \& Society, 25, 59-72. doi:10.1111/j.10990860.2009.00260.x

\section{Correspondance}

Émilie Myriam Roy

Courriel : emilie-myriam.roy.1@ulaval.ca

\section{Résumé}

La cyberintimidation est une forme de violence de plus en plus préoccupante pour la société et pour le milieu scolaire. Souvent commises dans Internet ou sur le téléphone cellulaire, les agressions ont des conséquences psychologiques, sociales et scolaires très graves chez les victimes, les agresseurs et les témoins, et il est important d'intervenir adéquatement. Cette étude vise à mieux connaître les types d'intervention utilisés par les enseignants auprès des élèves qui ont des comportements de cyberintimidation et à mieux comprendre les bases qui expliquent ces choix d'intervention. Des entrevues menées auprès de 10 enseignants des $7^{\mathrm{e}}, 8^{\mathrm{e}}$ et $9^{\mathrm{e}}$ années ont permis de relever leurs interventions les plus courantes pour gérer et traiter les comportements de cyberintimidation de leurs élèves, connaître leur perception de l'efficacité de ces pratiques et explorer les fondements théoriques sur lesquels ils s'appuient dans leurs choix d'intervention. L'analyse de 
leurs témoignages, mise en perspective avec la littérature scientifique sur le sujet, permet de proposer un nouveau modèle d'intervention auprès des auteurs de cyberintimidation. Après avoir confronté les résultats au modèle d'intervention d'Hinduja et Patchin (2009), les auteures suggèrent de nouvelles pistes d'intervention pour améliorer la formation des enseignants dans ce domaine.

Mots-clés : cyberintimidation, cyberintimidateurs, intervention des enseignants, pratiques éducatives

$$
\text { *** }
$$

\section{Abstract}

Cyberbullying is a form of online violence that is of growing concern in our society and in the school environment. In light of the fact that the aggressions, made on the Internet and on cellular phones, can have serious psychological, social and academic consequences for the victims, the bystanders and the cyberbullies, it is important to intervene appropriately. This study aims to understand teachers' perception of their interventions with students who cyberbully and the theoretical basis or concepts guiding these interventions. Semi-structured interviews, conducted with ten teachers of grades 7,8 and 9 , revealed the most popular reactions of teachers to their students' cyberbullying behaviours. This study also explores the basis influencing the teachers' choices of interventions and their perception of the effectiveness of these practices. The analysis of the teachers' accounts, put in perspective with a literature review, suggests ways to improve their training to address cyberbullying. A new intervention model is suggested.

Keywords : cyberbullying, cyberbullies, teachers' intervention, educational practices 